\title{
Petri nets for modelling and analysing trophic networks
}

\author{
Paolo Baldan ${ }^{1}$, Martina Bocci ${ }^{2}$, Daniele Brigolin ${ }^{2}$, Nicoletta Cocco $^{2}$, and \\ Marta Simeoni ${ }^{2}$ \\ 1 Dipartimento di Matematica, Università di Padova, Italy \\ baldan@math . unipd.it \\ 2 Dipartimento di Scienze Ambientali, Informatica e Statistica, \\ Università Ca' Foscari di Venezia, Italy \\ \{martina.bocci, brigo, cocco, simeoni\}@unive.it
}

\begin{abstract}
We consider trophic networks, a kind of networks used in ecology to represent feeding interactions (what-eats-what) in an ecosystem. We observe that trophic networks can be naturally modelled as Petri nets and this suggests the possibility of exploiting Petri nets for the analysis and simulation of trophic networks. Some preliminary steps in this directions and some ideas for future development are presented.
\end{abstract}

\section{Introduction}

Ecosystems are very complex systems constituted by biotic communities (populations of different species), abiotic components of the environment (like air, water, soil) and interactions among these (living and non-living) elements. A branch of ecology deals with the study of feeding relationships within ecosystems and represents them as networks of interacting compartments called trophic networks or food webs. Due to the common limited availability of experimental information, a static approach (the mass balance steady state approach) to the study of such networks has been developed as alternative to the dynamic description.

Complex networks of interacting entities are widely studied in computer science: computer networks, agent systems, and, in general, all concurrent and distributed systems fall into this category. Uncountably many formalisms and practical tools have been developed for the representation and analysis of interacting systems. This suggests the possibility of reusing models and techniques from computer science for the study of trophic networks.

This idea is pursued in [17], where the authors advocate the use of process calculi for ecological modelling. Their claim is that the compositionality properties of process calculi can be fruitfully exploited for a modular representation of complex ecosystems. Moreover, process calculi provide an individual based modelling and stochastic extensions.

In this paper we explore the use of another widely used model of concurrency, namely Petri nets $[19,11]$. Petri nets permit individual based modelling, they explicitly represent parallelism and dependencies among entities, they offer 
stochastic and continuous extensions and, as a major advantage, they enable a qualitative analysis of systems when dynamic information are not available. Many tools for systems visualisation, analysis and simulation are also available (see The Petri net World site [21]). In this paper we consider the representation and analysis techniques generally adopted for trophic networks and discuss the pros and cons of the application of Petri nets to this field.

The structure of the paper is as follows. In Section 2 trophic networks are introduced with a small case study related to the Venice lagoon. In Section 3 the main concepts in Petri nets used to model trophic networks are briefly recalled. In Section 4 we propose a simple application of Petri nets to the representation and analysis of trophic networks when dynamic information are not available. This is exemplified in the case study. Some conclusions and suggestions for further work are given in Section 5.

\section{Tropic Networks}

An ecosystem is a community of living organisms, such as plants, animals and microbes, in conjunction with the nonliving components of their environment, such as air, water and bioavailable organic matter (detritus), which interact as a system. A trophic network (or food web) is a representation of feeding interactions in an ecosystem, where the components are connected by binary links (what-eats-what). Food webs permit to represent and analyse the trophic structure and functioning of an ecosystem. This knowledge can be used to identify key species and to detect anthropogenic impacts, such as the effects of pollution, of physical disturbance, of resources exploitation, etc. Real trophic networks are very complex, hence models provide partial and abstract representations where, for instance, similar species are aggregated into groups with similar feeding behaviour. Model representation of a trophic network generally focuses on the fluxes of energy or biomass between nodes. Such fluxes are directional and generally encompass some very relevant organism-level processes, such as production, consumption, assimilation, predation, non-predatory mortality and respiration. An ecosystem is generally an open system, i.e. there are flows of material or energy between the system and the rest of the world. For this reason, when representing and analysing trophic networks, generally also the input and output flows are taken into account. Inputs can be primary production, immigration or incoming of detrital matter into the system, while outputs can be emigration, harvesting by humans and exit of detrital matter from the system. Some energy may be dissipated into heat (respiration) or some material may be degraded into its lowest energy form (detritus).

Knowledge on the species present in the studied ecosystem and on their feeding behaviour is a needed prerequisite for representing the trophic network. First of all it is necessary to single out the $n$ living and non-living compartments to be represented. A compartment can represent a population of a given species or of some aggregation of species with comparable feeding habits. For each compartment it is necessary to determine which other taxa are included in its diet, thus 


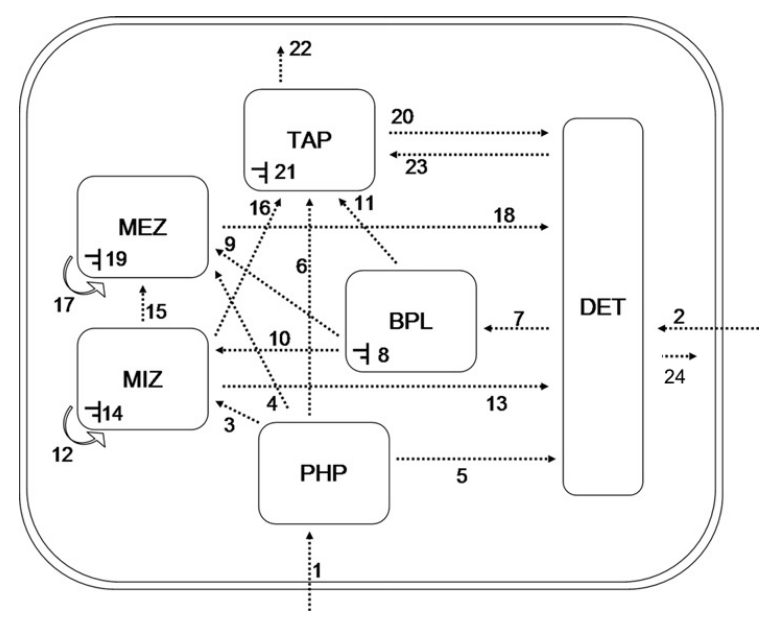

\begin{tabular}{|c|c|}
\hline \multicolumn{2}{|c|}{ Nocost. Flux } \\
\hline 1 & $\mathrm{CO}_{2} \rightarrow \mathrm{PHP}$ \\
\hline 2 & input $\rightarrow \mathrm{DET}$ \\
\hline 3 & $\mathrm{PHP} \rightarrow \mathrm{MIZ}$ \\
\hline 4 & $\mathrm{PHP} \rightarrow \mathrm{MEZ}$ \\
\hline 5 & $\mathrm{PHP} \rightarrow \mathrm{DET}$ \\
\hline 6 & $\mathrm{PHP} \rightarrow \mathrm{TAP}$ \\
\hline 7 & $\mathrm{DET} \rightarrow \mathrm{BPL}$ \\
\hline 8 & $\mathrm{BPL} \rightarrow \mathrm{CO}_{2}$ \\
\hline 9 & $\mathrm{BPL} \rightarrow \mathrm{MEZ}$ \\
\hline 10 & $\mathrm{BPL} \rightarrow \mathrm{MIZ}$ \\
\hline 11 & $\mathrm{BPL} \rightarrow \mathrm{TAP}$ \\
\hline 12 & $\mathrm{MIZ} \rightarrow \mathrm{MIZ}$ \\
\hline 13 & $\mathrm{MIZ} \rightarrow \mathrm{DET}$ \\
\hline 14 & $\mathrm{MIZ} \rightarrow \mathrm{CO}_{2}$ \\
\hline 15 & $\mathrm{MIZ} \rightarrow \mathrm{MEZ}$ \\
\hline 16 & $\mathrm{MIZ} \rightarrow \mathrm{TAP}$ \\
\hline 17 & $\mathrm{MEZ} \rightarrow \mathrm{MEZ}$ \\
\hline 18 & $\mathrm{MEZ} \rightarrow \mathrm{DET}$ \\
\hline 19 & $\mathrm{MEZ} \rightarrow \mathrm{CO}_{2}$ \\
\hline 20 & $\mathrm{TAP} \rightarrow \mathrm{DET}$ \\
\hline 21 & $\mathrm{TAP} \rightarrow \mathrm{CO}_{2}$ \\
\hline 22 & $\mathrm{TAP} \rightarrow$ Harvesting \\
\hline 23 & $\mathrm{DET} \rightarrow \mathrm{TAP}$ \\
\hline 24 & $\mathrm{DET} \rightarrow$ Export \\
\hline
\end{tabular}

Fig. 1. A trophic network $\mathcal{T}_{V}$ of the Venice Lagoon [4] (left) and its fluxes (right).

specifying the interactions among species or groups of species. These information determine the network topology, which already provides some relevant insights on the features of the ecosystem. It is normally represented as a directed graph where each node represents a compartment and each arc denotes an interaction between the source and target nodes. More precisely, an arc from node A to node $\mathrm{B}$ represents a flow of energy or biomass from A to B. A common convention is to depict dissipation for some node with an arc outgoing from the node and ending in the ground symbol of electrical circuits [20]. A quantity may be associated with each arc, representing the magnitude of biomass or energy flow or the relative occurrence of such a flow. The resulting graph is a directed weighted graph.

The graph of a simple planktonic trophic network of the Venice Lagoon, taken from [4], is shown in Figure 1 (left). Numbers on arrows indicate the fluxes, which are listed in Figure 1 (right). The compartments considered are phytoplankton (PHP), bacterioplankton (BPL), microzooplankton (MIZ), mesozooplankton (MEZ), R. philippinarum (TAP) and organic detritus (DET). The network provides a representation of the food items digested and assimilated by R. philippinarum (a marine bivalve mollusk), namely, green algae, cyanobacteria, diatoms, bacterioplankton, microzooplankton, and dead, dissolved, and/or particulate organic matter.

This trophic network has some peculiarities:

- dissipation (respiration) of PHP is not considered because the flow from $\mathrm{CO}_{2}$ to PHP models the net photosynthetic production, known from experimental data, i.e. the $\mathrm{CO}_{2}$ needed for respiration has been already subtracted; 
- flow from BPL to DET (mortality of BPL) is not considered because it is known to be negligible by experimental data;

- flows from TAP, MEZ and MIZ to DET include both natural mortality and production of faeces;

- flow from PHP to DET indicates only mortality, because PHP does not produce faeces;

- in the case of MIZ and MEZ cannibalism is represented by arrows exiting and entering in the same compartment (flows 12 and 17).

From the topology of the graph, or the corresponding adjacency matrix, some information about system behaviour can be derived. Clearly the adjacency matrix does not represent the information on weights of the interactions. For this reason various other matrices have been defined and used for analysis purposes, such as the matrix of dietary coefficients, the Leontief structure matrix, the total dependency matrix and many others which express different views of the network in relation to structural and quantitative dependencies among compartments [28]. The main advantage of a matrix representation of a trophic network is that linear algebra techniques can be applied and in fact matrix methods are the most used for static analysis of trophic networks (e.g. I-O modelling techniques for economics modified for ecosystems [28]).

To move from purely topological analysis of a trophic network to quantitative analysis, ecologists need quantitative data. Estimation of biomass and knowledge of several rates (e.g. production rate, consumption rate, respiration rate, etc.) are needed to quantify flows among compartments, together with quantitative knowledge about diet composition of each living compartment. Some information on primary production, specific consumption rates and diet compositions can be gained from field and laboratory studies but it is unfeasible to determine the magnitudes of all flows in the system directly. It becomes necessary, therefore, to estimate the magnitudes of some of them by indirect means. A helpful approach for estimating unknown flows consists in assuming the balance of inputs and outputs for each compartment. If a sufficiently long time period is considered, mass balance in each node of the network is a reasonable assumption because of the conservation of mass principle. Under the mass balance assumption, the system is represented as a steady state snapshot of energy flows, averaged over time. Different techniques are used for the trophic network reconstruction, that is to infer unspecified flows by solving the balance equations and satisfying the constraints among the flows in the system. The problem is generally underdetermined and an infinite number of solutions comply with the data set and the mass balance assumption. One technique is the Inverse Model (IM), which has been firstly applied to trophic network in [31] and it has become quite common among ecologists. IM combines mass balance equations, data equations and constraints on the flows expressed as inequalities. It finds a unique solution based on some optimisation criteria, for example by minimising the sum of squared flows, which corresponds to the most parsimonious solution. The package LIM implements linear inverse models in R [29]. Another freely available popular automated balancing routine that supports representation of 
trophic networks, estimation of unknown flows and ecological network analysis is Ecopath [6] and its evolutions Ecopath-Ecosym-Ecospace [7,8].

Several analyses on ecological networks have been defined in the last decades. Some of them are based only on the topology of the model, for instance determining food chain length, connectance and the presence of cycles. In a balanced model it is possible to study both qualitative and quantitative properties measured by global system status indexes such as degree of recycling [2], stability [16, 30], development [27], ascendency [28] and maturity [20]. Analysis of recycling is intended to characterise how the biomass or energy is reused in a trophic network. Such analysis requires the topology of pathways over which the medium is recycled, as well as the amounts of material cycling in each loop. In [28] the author proposes to do this into two steps: first all simple cycles in the network are identified, then cycled flows are separated from straight-through flows and a technique is proposed to identify and subtract them from the original network.

\section{Petri Nets}

Petri nets are a well known formalism originally introduced in computer science for modelling discrete concurrent systems. Petri nets have a sound theory and many applications which are not limited to computer science (see, e.g., [19] and [11] for surveys). A large number of tools have been developed for analysing Petri nets (see a list at the Petri Nets World site [21]).

We denote a basic Petri net by $N=\left(P, T, W, M_{0}\right)$, where $P=\left\{p_{1}, \ldots, p_{n}\right\}$ is the set of places, $T=\left\{t_{1}, \ldots, t_{m}\right\}$ is the set of transitions, $W:((P \times T) \cup$ $(T \times P)) \rightarrow \mathbb{N}$ is the weight function and $M_{0}$ is the initial marking of the net, an $n$-dimensional integer vector assigning to each place its initial number of tokens.

We write $t^{-}$for the pre-condition of a transitions $t$, namely the $n$-dimensional vector $t^{-}=\left(i_{1}, \ldots, i_{n}\right)$, where $i_{j}=W\left(p_{j}, t\right)$ for $j \in\{1, \ldots, n\}$. Sometimes it will be confused with its support, i.e., the set of places $\left\{p_{j} \mid i_{j}>0\right\}$. The post-condition $t^{+}=\left(o_{1}, \ldots, o_{n}\right)$ is defined dually.

The incidence matrix of a Petri net $N$, denoted by $\mathcal{A}_{N}$, is the $n \times m$ matrix which has a row for each place and a column for each transition. The column associated with transition $t$ is the vector $\left(t^{+}-t^{-}\right)^{T}$, which represents the marking change due to the firing of $t$.

Depending on the available information, Petri nets may permit to represent and study a system qualitatively, based only on the graph structure, as much as quantitatively or dynamically. An interesting structural analysis is based on the incidence matrix and it aims to determine the so-called invariants of the net. We focus here on T-invariants. Let $N$ be a Petri net, with $m$ transitions and $n$ places, a T-invariant (transition invariant) of $N$ is a multiset of transitions whose execution starting from a state will bring the system back to the same state, namely it is an $m$-dimensional vector in which each component represents the number of times that a transition should fire to take the net from a state $M$ back to $M$ itself. It can be obtained as a solution of the equation

$$
\mathcal{A}_{N} \cdot X=0 \text {, where } X=\left(x_{1}, \ldots, x_{m}\right)^{T} \text { and } x_{i} \in \mathbb{N} \text {, for } i \in\{1, \ldots, m\} \text {. }
$$


A T-invariant $X \neq 0$ indicates that the system can cycle on a state $M$ enabling the cycle. As discussed in $[13,18]$, T-invariants admit two possible interpretations. On the one hand, the components of a T-invariant represent a multiset of interactions (transitions) whose partially ordered execution reproduces a given initial state of the system (marking). On the other hand, the components of a T-invariant may be interpreted as the relative rates of interactions (transitions) which occur permanently and concurrently in a steady state. Minimal T-invariants of a finite Petri net, $N$, form a basis, $\mathcal{B}(N)$, for the set of semipositive T-invariant (Hilbert basis [24]). Any T-invariant can be obtained as a linear combination, with positive integer coefficients, of elements of the basis. Uniqueness of the basis $\mathcal{B}(N)$ makes it a characteristic feature of the net $N$.

Two subclasses of Petri nets will be of interest in the modelling of trophic networks [10]. A state machine Petri net is a Petri net where every arc has weight one and every transition has exactly one place in its pre- and post-condition. State machine Petri nets are conservative, namely the total number of tokens of the system remains invariant under the occurrence of transitions. A free choice Petri net is characterised by the fact that for any place $p$, either $p$ has at most one post-transition (i.e. no conflict) or it is the only pre-place of all its posttransitions. The class of state machine Petri nets is strictly included in the class of free choice Petri nets.

Petri nets supply an executable specification: in the case of basic Petri nets, we can play the token game, i. e. the non-deterministic firing of all the enabled transitions. More sophisticated and realistic models and simulations can be obtained through extended Petri net models. The most interesting in our context are Continuous Petri nets. In Continuous Petri nets [13] the state is no longer discrete. Places contain non-negative real numbers, called marks, usually interpreted as the concentration of the species represented by the place. The instantaneous firing of a transition is carried out like a continuous flow. The firing rate expresses the "speed" of the transformation from input to output places. The rate functions associated with transitions may follow, under simplifying assumptions, known kinetic equations such as the mass action equation.

\section{Petri Nets for Analysing Trophic Networks}

We start discussing how Petri nets can be used to model and analyse a trophic network. We assume to know only the species (or compartments) and their relations, which is the minimal knowledge generally available on a trophic network. As a running example, we consider the trophic network $\mathcal{T}_{V}$ of the Venice lagoon in Figure 1. We illustrate how to build corresponding Petri net models and discuss what we can obtain by applying some Petri net analysis techniques. We use the tools Snoopy [14], Charlie [15] and 4ti2 [1] for editing and analysing the Petri net models. 


\subsection{Modelling trophic networks with Petri nets}

Given a trophic network $\mathcal{T}$, a simple Petri net model can be immediately derived by replicating the topological structure of $\mathcal{T}$ in the Petri net. Recall that in the graph representation of $\mathcal{T}$ each species (or compartment) is a node and a relation between two species is a directed arc representing the flux between the two species.

A structural Petri net model of a trophic network $\mathcal{T}$ is the net $N_{s}(\mathcal{T})$ where

- any species (or compartment) becomes a place;

- any flow (relation) between two species $S_{1}$ and $S_{2}$ in $\mathcal{T}$, becomes a transition having $S_{1}$ as a pre-condition and $S_{2}$ as a post-condition.

- any outgoing flow from a species $S_{1}$ to the external environment (e.g., dissipation) in $\mathcal{T}$, becomes a transition with pre-condition $S_{1}$ and empty postcondition; similarly, any incoming flow from the environment to a species $S_{2}$, becomes a transition with empty pre-condition and post-condition $S_{2}$.

In absence of any information regarding the fluxes, all weights are set to one. Transitions corresponding to interactions among species are referred to as internal transitions, while those corresponding to interactions with the environment are referred to as interface transitions. Note that the structural Petri net model of a trophic network is a free choice Petri net and, when restricted to internal transitions, it is a state machine Petri net.

By applying the described construction to the running example $\mathcal{T}_{V}$ in Figure 1, we obtain a structural Petri net model which is depicted in Figure 2 (for the moment, please ignore the rates associated with transitions). The net includes six places (in yellow) representing the six compartments (DET, PHP, BPL, MIZ, MEZ, TAP) of the trophic network, and by as many transitions as the flows of biomass, to which we associate different colors to improve readability. More specifically, respiration flows (producing $\mathrm{CO}_{2}$ ) are represented by light blue transitions; defecation flows are represented by brown transitions; mortality flows are represented by purple transitions; input and export flows for DET, as well as the harvesting flow for TAP are represented by red transitions; predationprey flows are represented by white transitions.

Note that transitions PHP_CO2, representing respiration of PHP, and BPL_DET, representing BPL mortality in the Petri net model of Figure 2, do not have a direct match in the trophic network $\mathcal{T}_{V}$ of Figure 1 . This is due to the fact that, as already mentioned, $\mathcal{T}_{V}$ was simplified by taking into account also some experimental data. More precisely, the flow corresponding to PHP_CO2 was integrated in $\mathrm{CO} 2 \_\mathrm{PHP}$ (modelling $\mathrm{CO}_{2}$ needed for photosynthesis) and BPL_DET was considered irrelevant and thus omitted.

\subsection{Structural analysis of trophic networks modelled as Petri nets}

Since the structural Petri net model strictly adheres to the graph representation used by ecologists, it obviously enables the usual structural analyses for trophic networks, for example to determine food chains lengths and connectance. 
In addition, standard structural analyses for Petri nets can be used, like those based on T-invariants. The presence of T-invariants in a Petri net model of a trophic network is ecologically of interest as it can reveal the presence of steady states. The set of transitions involved in a T-invariant can be seen as a subsystem of the original system, whose equilibrium is autonomously maintained.

Given a trophic network $\mathcal{T}$, consider the set of semi-positive T-invariants of the structural Petri net model $N_{s}(\mathcal{T})$ and the corresponding Hilbert basis $\mathcal{B}\left(N_{s}(\mathcal{T})\right)$, consisting of the minimal T-invariants. According to the terminology in [13], we classify T-invariants into two groups:

- internal T-invariants, consisting of internal transitions only;

- I/O T-invariants, which include also interface transitions.

If we consider the elements of the basis, then for any such T-invariant $I=$ $\left(x_{1}, \ldots, x_{m}\right)$ we have $x_{i} \leq 1$ for all $i \in\{1, \ldots, m\}$, namely each transition occurs at most once and the invariant is a set rather than a proper multiset. Moreover, since $N_{s}(\mathcal{T})$, when restricted to the internal transitions, is a state machine, for any pair of transitions $t_{i}, t_{j}$ in the same invariant, whenever they share a place in the pre-condition or in the post-condition, they coincide. Therefore:

- Minimal internal invariants are simple cycles, involving only internal transitions.

- Minimal I/O invariants are acyclic paths, connecting two interface transitions.

In both cases we recover well-known concepts in trophic networks as presented, e.g., in [28]. The internal minimal T-invariants are Ulanowicz simple cycles, which are associated with the internal recycling of matter. The minimal I/O T-invariants are the Ulanowicz straight-through flows, which represent the way energy and matter are provided by the environment, used by the network and then (partially) released back to the environment. The correspondence is at the structural level and the quantities of fluxes are needed for Ulanowicz analyses.

In our case study, the structural Petri net model has an Hilbert basis consisting of 69 minimal T-invariants, nine are internal and sixty are I/O invariants. The internal T-invariants are shown in Table 1. The first two invariants describe the self-predation (cannibalism) of MEZ and MIZ. All the other T-invariants "traverse" the DET place, pointing out that Detritus is the way for recycling matter in this network. The I/O invariants start from source transitions CO2_PHP and input_DET and end in sink transitions PHP_CO2, BPL_CO2, MIZ_CO2, MEZ_CO2, TAP_CO2 and TAP_harvesting. They model trophic chains allowing for respiration of the various compartments and for input and output of matter.

\subsection{T-invariant based steady state}

In this section we refine the structural Petri net model of a trophic network, turning it into a continuous Petri net model. What we obtain closely resembles the representation of the trophic network usually adopted by ecologists, where 


\begin{tabular}{|cl|}
\hline Inv no. Transitions \\
1 & MEZ_MEZ \\
2 & MIZ_MIZ \\
3 & DET_TAP; TAP_DET \\
4 & DET_BPL; BPL_DET \\
5 & DET_BPL; BPL_MEZ; MEZ_DET \\
6 & DET_BPL; BPL_MIZ; MIZ_DET \\
7 & DET_BPL; BPL_TAP; TAP_DET \\
8 & DET_BPL; BPL_MIZ; MIZ_MEZ; MEZ_DET \\
9 & DET_BPL; BPL_MIZ; MIZ_TAP; TAP_DET \\
\hline
\end{tabular}

Table 1. Internal minimal T-invariants of the structural Petri net model of $\mathcal{T}_{V}$.

the system is at a steady state and the input and output flows in all the compartments are balanced (the mass balance assumption). The choice of considering a continuous extension is motivated by the fact that we are modelling fluxes of biomass which better correspond to continuous fluxes.

The continuous Petri net model is still derived only from the network topology by exploiting the minimal T-invariants in a way similar to what is done in [22] for Time Petri nets. A first observation is in order.

Remark 1. In the structural Petri net model of a trophic network $N_{s}(\mathcal{T})$ any place has typically at least one incoming and one outgoing transition, otherwise the place would unnaturally correspond to a compartment with monotonically increasing or decreasing content. Under this assumption, $N_{s}(\mathcal{T})$ is covered by $T$-invariants, namely each transition in the Petri net belongs to at least one minimal T-invariant. In fact, when we exclude interface transitions $N_{s}(\mathcal{T})$ is a state machine, hence for any transition, if we follow the predecessors and successors we will get back to the transition itself (internal T-invariant) or to an interface transition on both sides (I/O T-invariant).

In order to associate rates with the transitions, we assume that each subsystem corresponding to a minimal T-invariant

1. is active and

2. performs all its transitions once per time unit.

The assumption that all minimal subsystems of an ecosystem are active is quite reasonable from an ecological viewpoint. On the contrary the assumption that all subsystems perform all their transitions exactly once per time unit is rather strong and unrealistic. This is the simplest choice which can be taken in absence of further information on the ecosystem. When additional knowledge is available, it could be integrated in the model, as shown in the next section.

Let us consider the structural Petri net model $N_{s}(\mathcal{T})$ of a trophic network $\mathcal{T}$ as described in Section 4.1 and its Hilbert basis $\mathcal{B}\left(N_{s}(\mathcal{T})\right)$. According to the assumptions above, the rate of a transition $t$ should depend on the number of minimal invariants in which $t$ occurs. Then, for the trophic network $\mathcal{T}$, we define the simple continuous Petri net model $N_{c}(\mathcal{T})$ as the continuous Petri net obtained by considering the structural model $N_{s}(\mathcal{T})$ as underlying Petri net and by associating to each transition $t$ a constant rate given by: 


$$
\operatorname{rate}(t)=\left|\left\{I_{i} \mid I_{i} \in \mathcal{B}\left(N_{s}(\mathcal{T})\right) \wedge t \in I_{i}\right\}\right| .
$$

With such rates, all the transitions in all the invariants in $N_{c}(\mathcal{T})$ are performed once in one time unit and the system is in a steady state. Moreover, since all transition arcs are 1-weighted, rates and flows per time unit coincide.

Remark 2. The continuous Petri net model of a trophic network satisfies the mass balance assumption, namely, for all compartments the sum of ingoing and outgoing fluxes coincide. This is an immediate consequence of the fact that minimal T-invariants are simple cycles or paths. Hence, given a place $p$, for any invariant $I_{i}$ that "crosses" place $p$, one token is added to $p$ by a transition in $I_{i}$ and one token is consumed by another transition in $I_{i}$, namely the flux flowing through $p$ via $I_{i}$ is balanced. This holds for any invariant crossing $p$ and for any $p$. Therefore, the input and output fluxes coincide for any place of the network.

In the simple continuous model $N_{c}(\mathcal{T})$, the system is represented in a steady state, with the fluxes of biomass balanced in all compartments. This corresponds closely to the ecologists representation of a trophic network as a snapshot of the system at steady state. Note that the continuous Petri net model $N_{c}(\mathcal{T})$, despite the fact that it makes explicit some additional features, is still based only on the topology of $\mathcal{T}$ : biomasses do not play a role in the definition of the rates.

For our case study, the continuous Petri net model resulting from the construction outlined above is shown in Figure 2, where each transition have an associated rate. Note that all places are balanced. We would like to validate our simple continuous model by considering some basic ecological processes and check their plausibility from an ecological point of view. For each compartment we compute the throughput, namely the total amount of flux flowing per unit of time, in order to measure the degree of activity of the compartment. Besides we compute food consumption (total amount of ingested food per time unit), food assimilation (amount of ingested food minus amount of faeces, per time unit), respiration and mortality as percentages of the consumption. Table 2 shows the throughputs, the assimilation and respiration values as resulting from the model compared with those found in the literature.

The values derived from the simple continuous model are quite interesting. Considering the throughput, the various compartments are ordered as follows:

$$
\text { DET }>\text { PHP }>\text { BPL }=\text { TAP }>\text { MIZ }>\text { MEZ } .
$$

We may distinguish two main groups: lower trophic level compartments (DET, PHP and BPL), having higher throughput, and higher trophic level compartments (TAP, MIZ and MEZ), having lower throughput. This is coherent with the general knowledge on metabolic and growth rates of the two different groups of compartments under consideration.

Assimilation of the top compartment TAP is just over the maximum indicated in the literature, while assimilation requirements for MEZ and MIZ are perfectly met. However, MEZ assimilation is close to the lower bound of the indicated range. This is due to the fact that MEZ is a top level compartment 


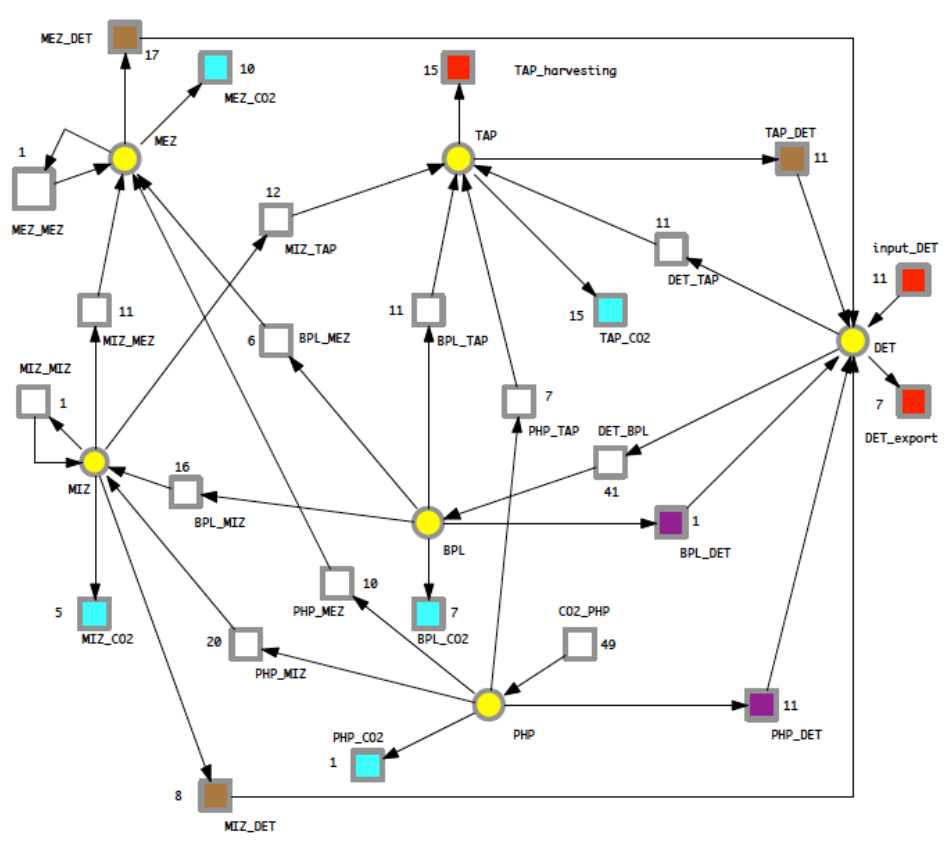

Fig. 2. Continuous Petri net model for the case study

in the network and no predators are modelled for it. This is a quite unrealistic assumption: in natural systems MEZ are actually preyed by other species, like fishes. By adding an external predation on MEZ, we found that its assimilation becomes close to TAP and MIZ assimilation values.

Concerning respiration, TAP and MEZ satisfy the constraints found in the literature, while MIZ and BPL are slightly below the indicated value. Respiration of PHP is instead largely below the lower bound of the indicated range. The low respiration flows for MIZ, BPL and PHP is caused by the fact that there are only a few I/O minimal invariants involving these compartments. This is a misbehaviour of the simple continuous model, that must be somehow overcome.

Concerning mortality, for BPL it is irrelevant and this is in accordance with experimental data (see discussion in Section 2). Mortality of PHP is instead quite high: this is probably due to the fact that some PHP grazers, like fishes usually occurring in lagoon systems, are not modelled.

On the whole, the continuous Petri net model realistically reproduces the main processes of the trophic network considered in the case study. Even if it based only on the network topology, it allows for deriving some quantitative information on trophic network flows, which are coherent with results of experimental measures taken in natural ecosystems. Moreover, the quantitative validation shows that the model is somehow incomplete, signalling that two further predation fluxes, one for MEZ and one for PHP, should be represented in the model. 


\begin{tabular}{|c|c|l|l|}
\hline Compartment & throughput & Literature values & Model values \\
\hline TAP & 41 & $\begin{array}{l}{[25] \text { Respiration } \geq 20 \%} \\
{[25] 37 \% \leq \text { Assimilation } \leq 70 \%}\end{array}$ & $\begin{array}{l}\text { Respiration }=36 \% \\
\text { Assimilation }=73 \% \\
\text { Defecation and Mortality }=27 \%\end{array}$ \\
\hline MEZ & 28 & $\begin{array}{l}{[12] \text { Respiration } \geq 20 \%} \\
{[23,9] 40 \% \leq \text { Assimilation } \leq 80 \%}\end{array}$ & $\begin{array}{l}\text { Respiration }=37 \% \\
\text { Assimilation }=39 \% \\
\text { Defecation and Mortality }=61 \%\end{array}$ \\
$\begin{array}{l}\text { Respiration }=14 \% \\
\text { Assimilation }=78 \% \\
\text { Defecation and Mortality }=22 \%\end{array}$ \\
\hline MIZ & 37 & $\begin{array}{l}{[12] \text { Respiration } \geq 20 \%} \\
{[23,9] 40 \% \leq \text { Assimilation } \leq 80 \%}\end{array}$ & $\begin{array}{l}\text { Respiration = 17\% } \\
\text { Assimilation = Consumption } \\
\text { Mortality =2,4\% }\end{array}$ \\
\hline BPL & 41 & $\begin{array}{l}{[26,5] \text { Respiration } \geq 20 \%} \\
\text { Assimilation = Consumption }\end{array}$ & $\begin{array}{l}\text { Respiration }=2 \% \\
\text { Assimilation }=\text { Consumption } \\
\text { Mortality }=22 \%\end{array}$ \\
\hline PHP & 49 & $\begin{array}{l}{[32,3] 10 \% \leq \text { Respiration } \leq 30 \%} \\
\text { Assimilation = Consumption }\end{array}$ \\
\hline DET & 58 & not relevant relevant \\
\hline
\end{tabular}

Table 2. Literature values and measured values for the continuous Petri net model of the case study.

\subsection{Introducing ecological constraints in the Petri net model}

In the previous section we underlined some misbehaviours of the simple continuous Petri net model. These are somehow expected since the model is only based on the topology of the system and it relies on the strong assumption that all subsystems proceed at the same speed. In order to adjust the model and make it closer to the real trophic network, one can follow two directions:

1. Drop the assumption that all the subsystems perform their path exactly once in one time unit and "speedup" some subsystems.

2. Use additional knowledge on the trophic network besides the topology, such as the metabolism of the species or their diet, and impose some constraints on the rates of the corresponding transitions.

We next examine more closely these two alternatives and apply them to our case study.

Speeding-up subsystems. Recall that any linear combination of minimal T-invariants is a T-invariant and a possible steady state of the network. Let us consider a generic linear combination of all minimal T-invariants:

$$
\sum_{I_{i} \in \mathcal{B}\left(N_{c}(\mathcal{T})\right)} k_{i} I_{i}, \quad k_{i} \in \mathbb{R}
$$

The simple continuous model $N_{c}(\mathcal{T})$ corresponds to a steady state given by a linear combination of all the minimal T-invariants where all the $k_{i}$ are set to one. The refined continuous Petri net model, $N_{c s}(\mathcal{T})$, is obtained from $N_{c}(\mathcal{T})$ by dropping the assumption that all subsystems have the same speed and setting the invariants constants $k_{i}$ to values possibly greater than one. In $N_{c s}(\mathcal{T})$ the rate associated with each transition is generalised to:

$$
\operatorname{rate}(t)=\sum_{I_{i} \in \mathcal{B}\left(N_{s}(\mathcal{T})\right), t \in I_{i}} k_{i}
$$




\begin{tabular}{|l|l|l|c||l|l|l|c|l|l|l|c|}
\hline No. & Transition & rate & $\begin{array}{c}\text { rate after } \\
\text { speedup }\end{array}$ & No. & Transition & rate & $\begin{array}{c}\text { rate after } \\
\text { speedup }\end{array}$ & No. & Transition & rate & $\begin{array}{c}\text { Tater } \\
\text { speedup }\end{array}$ \\
\hline 1 & CO 2 _PHP & 49 & 60 & 10 & BPL_MIZ & 16 & 17 & 19 & MEZ_CO & 10 & 10 \\
2 & input_DET & 11 & 14 & 11 & BPL_TAP & 11 & 11 & 20 & TAP_DET & 11 & 11 \\
3 & PHP_MIZ & 20 & 22 & 12 & MIZ_MIZ & 1 & 1 & 21 & TAP_CO 2 & 15 & 15 \\
4 & PHP_MEZ & 10 & 10 & 13 & MIZ_DET & 8 & 8 & 22 & TAP_Harv. & 15 & 15 \\
5 & PHP_DET & 11 & 11 & 14 & MIZ_CO & 5 & 8 & 23 & DET_TAP & 11 & 11 \\
6 & PHP_TAP & 7 & 7 & 15 & MIZ_MEZ & 11 & 11 & 24 & DET_Export & 7 & 7 \\
7 & DET_BPL & 41 & 44 & 16 & MIZ_TAP & 12 & 12 & 25 & PHP_CO 2 & 1 & 10 \\
8 & BPL_CO & 7 & 9 & 17 & MEZ_MEZ & 1 & 1 & 26 & BPL_DET & 1 & 1 \\
9 & BPL_MEZ & 6 & 6 & 18 & MEZ_DET & 17 & 17 & & & & \\
\hline
\end{tabular}

Table 3. Rates of the continuous Petri net model before and after speedup.

The refined continuous Petri net model $N_{c s}(\mathcal{T})$ still represents the trophic network at a steady state and with all compartments balanced, since the input and output fluxes are balanced in each place for each minimal T-invariant.

We applied this idea to the case study and speed up the invariants involving respiration of PHP, BPL and MIZ, since the respiration flows of these compartments do not satisfy the ranges indicated in the literature (see Table 2). The new rates for the transitions are shown in Table 3.

Concerning PHP, it receives in input $\mathrm{CO}_{2}$ and partially release it for respiration. The unique I/O T-invariant for this process is $\{\mathrm{CO} 2$ _PHP; PHP_CO2 $\}$. By speeding up this invariant to run ten times per unit of time, the respiration flow for PHP becomes the $16 \%$ of its total consumption, within the range indicated by the literature (see Table 2).

Concerning BPL, it is fed by the Detritus and part of the ingested food is used for respiration. The invariants involving transition BPL_CO2 are $\{\mathrm{CO} 2 \mathrm{PHP}$; PHP_DET; DET_BPL; BPL_CO2\} and \{input_DET; DET_BPL; BPL_CO2\}. By allowing the second invariant to run three times per unit of time, respiration of BPL become the $20 \%$ of its total consumption.

Concerning MIZ, we could speedup the invariants involving MIZ_CO2, namely \{CO2_PHP; PHP_DET; DET_MIZ; MIZ_CO2\} and \{input_DET; DET_MIZ; BPL_MIZ\}. By allowing them to run three and two times per unit of time, respectively, respiration of BPL becomes the $20 \%$ of its total consumption. Assimilation of BPL becomes the $80 \%$ of the consumption, still in the range indicated in Table 2.

Including constraints in the model. The second alternative for improving the model consists in "embedding" into the continuous Petri net model of the trophic network some available information regarding the metabolism of the species or their diet. We work under the simplifying assumption that flux constraints imposed on the model are linear. This assumption is generally satisfied by the constraints on metabolic fluxes and on the diet partitions. For our case study, some metabolic constraints taken from the literature are given in Table 2.

We define a continuous Petri net model which structurally coincides with $N_{s}(\mathcal{T})$ and whose transition rates satisfy a set of linear inequalities. As in the previous cases, the transition rates are derived from the "speed" $k_{i}$ of each minimal invariant, but now we are interested only in invariants that satisfy the 
constraints. These can be obtained as solutions of a system of inequalities

$$
\begin{aligned}
& A_{N} \cdot X=0 \\
& C \cdot X \geq 0
\end{aligned}
$$

where $A_{N}$ is the incidence matrix of $N_{s}(\mathcal{T})$. We can consider the minimal such T-invariants, referred to as the constrained Hilbert basis $\mathcal{B}_{C}\left(N_{s}(\mathcal{T})\right)$, so that any solution of (1) will be a linear combination of elements in $\mathcal{B}_{C}\left(N_{s}(\mathcal{T})\right)$.

A continuous Petri net model $N_{c}(\mathcal{T}, \mathcal{C})$ for the trophic network $\mathcal{T}$ satisfying the constraints $\mathcal{C}$ is defined as follows. The underlying Petri net is $N_{s}(\mathcal{T})$ and each transition $t$ is associated with a constant rate:

$$
\operatorname{rate}(t)=\left|\left\{I_{i}: I_{i} \in \mathcal{B}_{C}\left(N_{s}(\mathcal{T})\right) \wedge t \in I_{i}\right\}\right| .
$$

In this way each transition in each constrained invariant $I_{i}$ in $\mathcal{B}_{C}\left(N_{s}(\mathcal{T})\right)$ can be performed once in one time unit.

When applied to our case study, this approach produces a linear system of equalities and inequalities, where the inequalities express the literature knowledge summarised in Table 2. By considering only the inequalities given by the lower bounds, the constrained Hilbert basis contains 349 minimal invariants. The induced rate constants for the extended network automatically satisfy the given ecological constraints.

The two approaches could be combined, by determining the constrained invariants and by setting for them possibly different speeds.

Simulations on continuous models with constant rates do not provide meaningful information. Some hints on how to further refine the model to do simulation analyses are given in the conclusions.

\section{Conclusions and Future Work}

In this paper we explored the use of Petri nets for representing and analysing trophic networks and our preliminary results are encouraging. A trophic network naturally translates into a structural Petri net model which allows for recovering classical trophic networks concepts and analyses. The structural model can be refined into a continuous Petri net model that closely resembles the representation of the trophic network usually adopted by ecologists, where the system is at a steady state and the input and output flows are balanced in all the compartments. Despite the fact that the Petri net models proposed are still simplistic (in particular, the continuous models have constant rates, independent of the masses), in our case study of the Venice lagoon, the analysis of the continuous Petri net model shows that it realistically reproduces the main ecological processes. Furthermore, it shows that the continuous Petri net model can be fruitfully used for an early stage validation of the trophic network under study. Two refinements of the continuous Petri net are considered: the first is based on a fine tuning of the speed of the minimal T-invariants, while the second one is based on a systematic embedding of some ecological knowledge expressed as linear inequalities into the calculation of the Hilbert basis. This however might have 
scalability problems, since the constraints increase the size of the Hilbert basis, and the problem of determining the Hilbert basis is already in EXPSPACE.

Future work deals with making the Petri net model more realistic and dynamic, by adding biomass information on compartments. The knowledge of biomasses at a steady state can, in fact, be used to derive constants for a continuous model governed, e.g., by the mass action equation. We believe that introducing rates dependent on biomasses could allow for interesting simulations, describing, not only the steady state but also the transient behaviour leading to such state. Additionally, on such model perturbations of the biomasses and of the speed of the various interactions could be used for performing what-if analyses.

Acknowledgements. We are grateful to Monika Heiner and Andrea Marin for many inspiring discussions.

\section{References}

1. 4ti2 team. 4ti2 - a software package for algebraic, geometric and combinatorial problems on linear spaces. Available at www.4ti2.de.

2. S. Allesina and R. E. Ulanowicz. Cycling in ecological networks: Finn's index revisited. Computational Biology and Chemistry, 28:227-233, 2004.

3. R.S.K. Barnes and R.N. Hughes. An introduction to Marine Ecology. Wiley, 1999.

4. D. Brigolin and R. Pastres. Influence of intra-seasonal variability of metabolic rates on the output of a steady-state food web model. In Jordán F. and Jørgensen S.E., editors, Models of the Ecological Hierarchy: From Molecules to the Ecosphere, Developments in Environmental Modelling, pages 165-179. Elsevier, 2012.

5. C.A. Carlson, P.A. Del Giorgio, and G.J. Herndl. Microbes and the dissipation of energy and respiration: from cells to ecosystems. Oceanography, 20(2):89-100, 2007.

6. V. Christensen. Ecopath a software balancing steady-state models and calculating network characteristics. Ecological modelling, 61:169-185, 1992.

7. V. Christensen and C. J. Walters. Ecopath with Ecosim: methods, capabilities and limitations. Ecological modelling, 172(2):109-139, 2004.

8. V. Christensen, C. J. Walters, and D. Pauly. Ecopath with ecosim: a users guide. Fisheries Centre, University of British Columbia, Vancouver, 154, 2005.

9. R.J. Conover. Factors affecting the assimilation of organic matter by zooplankton and the question of superfluous feeding. Limnology and Oceanography, 11(3):346$354,2003$.

10. J. Desel and J. Esparza. Free Choice Petri Nets. Cambridge University Press, 2005.

11. J. Esparza and M. Nielsen. Decidability issues for Petri Nets - a survey. Journal Inform. Process. Cybernet. EIK, 30(3):143-160, 1994.

12. Vézina A. F. and M. L. Pace. An inverse model analysis of planktonic food webs in experimental lakes. Canadian Journal of Fisheries and Aquatic Sciences, 51(9):2034-2044, 1994.

13. M. Heiner, D. Gilbert, and R. Donaldson. Petri Nets for Systems and Synthetic Biology. In Proc. of SFM'08, volume 5016 of LNCS, pages 215-264. Springer, 2008. 
14. M. Heiner, M. Herajy, F. Liu, C. Rohr, and M. Schwarick. Snoopy a unifying Petri net tool. In Proc. of Petri Nets 2012, volume 7347 of LNCS, pages 398-407. Springer, 2012.

15. M. Heiner, M. Schwarick, and J. Wegener. Charlie an extensible petri net analysis tool. In Proc. of Petri Nets 2015, LNCS. Springer, 2015. to appear.

16. R. E. Heymans, J. J. Ulanowicz and C. Bondavalli. Network analysis of the south florida everglades graminoid marshes and comparison with nearby cypress ecosystems. Ecological Modelling, 149:5-23, 2002.

17. F. Jordán, M. Scotti, and C. Priami. Process algebra-based computational tools in ecological modelling. Ecological Complexity, 8(4):357-363, 2011.

18. I. Koch and M. Heiner. Petri nets. In B. H. Junker and F. Schreiber, editors, Analysis of Biological Networks, Book Series in Bioinformatics, pages 139-179. Wiley, 2008.

19. T. Murata. Petri Nets: Properties, Analysis, and Applications. Proceedings of IEEE, 77(4):541-580, 1989.

20. E.P. Odum. The strategy of ecosystem development. Science, 164(3877):262-270, 1969.

21. Petri nets tools. http://www.informatik.uni-hamburg.de/TGI/PetriNets/tools.

22. L. Popova-Zeugmann, M. Heiner, and I. Koch. Timed Petri Nets for modelling and analysis of biochemical networks. Fundamenta Informaticae, 67:149-162, 2005.

23. Parsons T. R., Takahashi M., and Hargrave B. Biological oceanographic processes. Pergamon Press, 1984.

24. A. Schrijver. Theory of linear and integer programming. Interscience series in discrete mathematics and optimization. Wiley, 1999.

25. I. Sorokin and O. Giovanardi. Trophic characteristics of the manila clam. ICES Journal of Marine Science, 52(5):853-862, 1995.

26. Reinthaler T., Winter C., and Herndl G. J. Relationship between bacterioplankton richness, respiration, and production in the Southern North Sea. Applied and environmental microbiology, 5(7):2260-2266, 2005.

27. R. E. Ulanowicz. A phenomenological perspective of ecological development. Aquatic Toxicology and Environmental Fate, 9:73-81, 1986.

28. R. E. Ulanowicz. Quantitative methods for ecological network analysis and its application to coastal ecosystems. Treatise on Estuarine and Coastal Science, 9:35-57, 2011.

29. D. van Oevelen, K. van den Meersche, F. R. Meysman, K. Soetaert, J. Middelburg, and A. Vézina. Quantifying Food Web Flows Using Linear Inverse Models. Ecosystems, 13:32-45, 2010.

30. M. Vasconcellos, S. Mackinson, K. Sloman, and D. Pauly. The stability of trophic mass-balance models of marine ecosystems: a comparative analysis. Ecological Modelling, 100:125-134, 1997.

31. A.F. Vézina and T. Platt. Food web dynamics in the ocean. I. Best-estimates of flow networks using inverse methods. Marine Ecology - Progress Series, 42:269-287, 1988.

32. R. G. Wetzel. Limnology. Lake and River Ecosystems. Elsevier, 2001. 